\title{
Successful treatment of a prolactinoma with the antipsychotic drug aripiprazole
}

\author{
Ilse C A Bakker1, Chris D Schubart² and Pierre M J Zelissen'1 \\ 1Department of Internal Medicine, Section of Endocrinology, University Medical Center Utrecht, \\ Utrecht, The Netherlands and 2Department of Psychiatry, Tergooi Hospital, Hilversum, The Netherlands
}

Correspondence should be addressed to P M J Zelissen

Email

P.M.J.Zelissen@umcutrecht.nl

\section{Summary}

In this report, we describe a female patient with both prolactinoma and psychotic disorder who was successfully treated with aripiprazole, a partial dopamine 2 receptor agonist. During the follow-up of more than 10 years, her psychotic symptoms improved considerably, prolactin levels normalised and the size of the prolactinoma decreased. This observation may be of clinical relevance in similar patients who often are difficult to treat with the regular dopaminergic drugs.

\section{Learning points:}

- Prolactinoma coinciding with psychosis can represent a therapeutic challenge.

- In contrast to many other antipsychotic drugs, aripiprazole is associated with a decrease in prolactin levels.

- Aripiprazole can be a valuable pharmaceutical tool to treat both prolactinoma and psychosis.

\section{Background}

Prolactinomas are among the most frequently occurring pituitary adenomas. The vast majority of these adenomas respond favourably to medical therapy with dopaminergic drugs leading to normoprolactinaemia and regression of adenoma size (1). Bromocriptine, quinagolide and cabergoline are the most frequently used dopaminergic drugs in the treatment of prolactinomas. The most common side effects are nausea, orthostatic hypotension and headache. Also the occurrence of CSF rhinorrhoea after medical treatment with dopamine agonists for macroprolactinoma has been reported (2). Psychiatric adverse effects may also occur and include insomnia, nightmares, hallucinations and even psychosis (3).

The treatment of a patient with prolactinoma and a concomitant psychotic disorder poses a particular challenge: psychotic symptoms are mostly treated with dopamine receptor $\mathrm{D}_{2}$ antagonists and the majority of antipsychotic agents can cause marked increases in prolactin levels as adverse events, which is particularly undesirable in patients with prolactinoma. Simultaneously, treatment with dopamine agonists may aggravate psychotic symptoms (4) since increased dopaminergic activity plays a central role in the pathophysiology of psychosis.

Aripiprazole is a relatively novel antipsychotic drug. In contrast to most other antipsychotics, aripiprazole is not associated with an increase in prolactin levels and may even result in a decrease in prolactin secretion (5). This rather unique property of aripiprazole could make this a suitable drug for the treatment of a patient with a prolactinoma and concomitant psychosis.

This case report describes a patient with a prolactinoma who experienced psychotic symptoms as side effects of treatment with dopamine receptor agonists. Treatment with aripiprazole, however, resulted in a favourable response to both psychotic symptoms and prolactinoma. 
Because of the long-time follow-up of more than 10 years, the effect of recurrent initiation and interruption of aripiprazole on prolactin levels are well illustrated.

\section{Case presentation}

In 2001, a 30-year-old female was referred to an internist in a local hospital because of amenorrhea and headaches since 2 years. She had a psychiatric history of depressive symptoms since 1995 and a postnatal depression in 1997.

Based on an elevated prolactin level of $2.25 \mathrm{IU} / \mathrm{L}$ (reference range $0.10-0.95 \mathrm{IU} / \mathrm{L}$ ) and a pituitary microadenoma of $8 \mathrm{~mm}$ in diameter on MRI (Fig. 1), she was diagnosed with microprolactinoma. Treatment with cabergoline and later quinagolide was initiated. Her prolactin levels normalised, followed by resumption of normal menses and disappearance of headaches. After 1 year, the adenoma size had decreased to $6 \mathrm{~mm}$ on MRI. However, the patient started to experience side effects such as emotional lability, insomnia and depressive symptoms during the use of both drugs. She discontinued the medication and the side effects resolved but prolactin levels increased again.

In February 2004, the patient was subsequently referred to our department for further treatment. At that time, she had stopped the use of cabergoline 4 weeks earlier. She did not use any other medication and experienced no psychiatric symptoms.

\section{Investigation}

The prolactin level was $2.99 \mathrm{IU} / \mathrm{L}$. Analysis of the other pituitary axes was normal. MR imaging showed a $6 \mathrm{~mm}$ pituitary microadenoma.

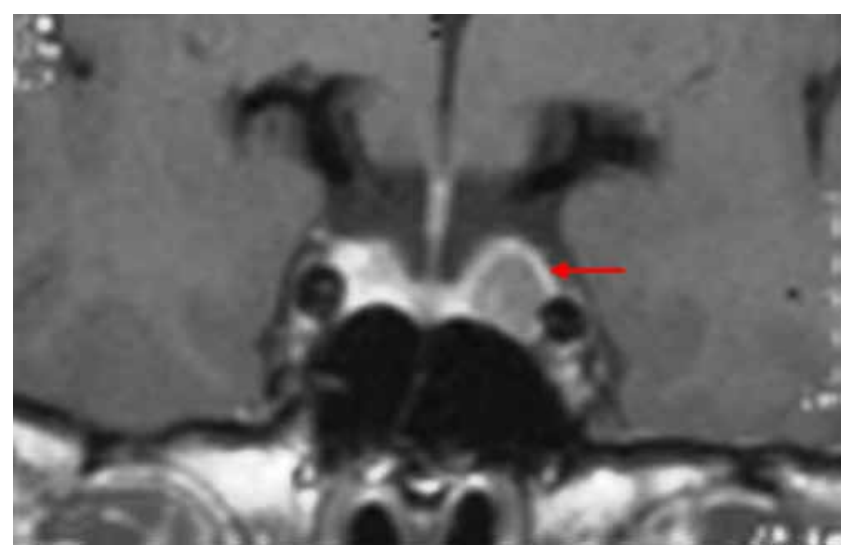

Figure 1

MRI scan of the sellar region showing a microadenoma on the left side of the pituitary gland, next to the carotid artery.

\section{Treatment}

Initially, the patient had a near-normal menstrual cycle. Therefore, we decided not to treat the prolactinoma for the time being and observe the course of the prolactin levels, the menstrual cycles and her mental condition. Six months later, the prolactin level had increased to $5.1 \mathrm{IU} / \mathrm{L}$ and the amenorrhea had recurred. Despite no use of medication, she had experienced episodes of depression and other psychiatric symptoms. We assumed there was no clear relationship between the use of dopaminergic medication and her psychiatric symptoms. We initiated treatment with a low dose ( $2.5 \mathrm{mg}$ twice daily) of bromocriptine.

\section{Outcome and follow-up}

Shortly after the start of this treatment, she developed visual, olfactory and tactile hallucinations and persecutory delusions and had to be admitted to a psychiatric hospital. Treatment with aripiprazole $7.5 \mathrm{mg}$ daily was initiated. On admission, her prolactin level was 3.0IU/L. One month after the start of the treatment with aripiprazole, the prolactin value had normalised to $0.63 \mathrm{IU} / \mathrm{L}$. Psychotic symptoms improved quickly with the use of aripiprazole. On MRI in 2005, the size of adenoma had decreased to $3 \mathrm{~mm}$ in diameter.

In August 2006, the patient became pregnant and temporarily stopped taking aripiprazole; however, a miscarriage occurred after 5 weeks. An MRI in 2006 showed an unchanged size of adenoma. Between 2006 and 2008, the prolactin values remained stable.

In May 2008, the patient became pregnant again and aripiprazole was discontinued. In January 2009, she started with haloperidol because of a deteriorated psychiatric condition. After delivery in February 2009, she switched back to aripiprazole. The patient decided to stop taking aripiprazole in mid-2009 because she experienced lethargy and feelings of agitation. A manic episode occurred and haloperidol was restarted. While using haloperidol, the serum prolactin values increased to $1.58 \mathrm{IU} / \mathrm{L}$. The MRI in mid-2009 showed an unchanged adenoma size.

The patient became pregnant again in June 2010. A new MRI did not show an increase in adenoma size.

Two years later, she developed more headaches, amenorrhea and an increase in depressive feelings, besides psychotic symptoms. Aripiprazole was restarted, initially together with haloperidol. Two weeks after the start of aripiprazole, her menses returned. Since July 2012, she has been using a low dose ( $3.75 \mathrm{mg}$ per day) of aripiprazole only, with a satisfying effect on both her psychiatric symptoms and the prolactin levels. Figure 2 shows an 


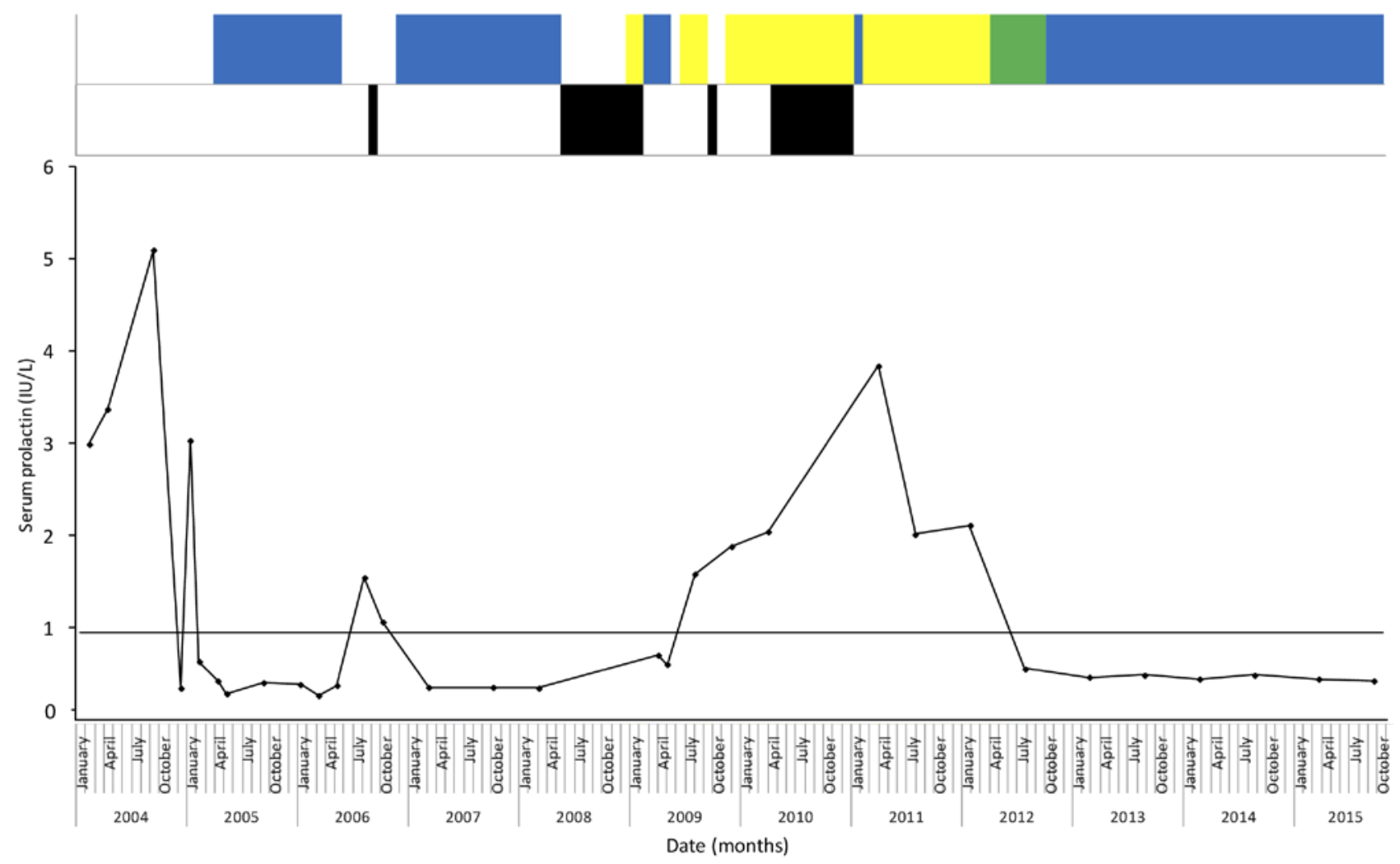

Figure 2

Overview of used antipsychotics (aripiprazole and haloperidol), episodes of pregnancy and prolactin values since the start of treatment from 2004 until 2014. The lower horizontal line indicates the upper limit of the reference range of serum prolactin ( $0.95 \mathrm{IU} / \mathrm{L})$. The blue area indicates the use of aripiprazole, the yellow area the use of haloperidol and the green shaded area the combination of aripiprazole and haloperidol. Pregnancy is indicated with the black areas.

overview of the prolactin levels, medications used and episodes of pregnancy between 2004 and 2014.

\section{Discussion}

This case report illustrates the prolactin lowering effect of aripiprazole in a patient with psychiatric disease and a concomitant prolactinoma during an extensive period of follow-up. Long-term normoprolactinaemia was observed during the use of aripiprazole as monotherapy. Every episode of recurrent hyperprolactinaemia in this patient was caused by special circumstances such as pregnancy, interruption of the use of aripiprazole or the addition of other psychiatric medication such as haloperidol.

The quinolone derivative and second-generation antipsychotic (SGA) aripiprazole was introduced in the late 1990s (6), and was found to be an effective treatment of schizophrenia and other psychotic disorders with a relatively low incidence of side effects that are associated with the use of many other antipsychotics such as weight gain, dyslipidaemia and extrapyramidal symptoms.
Most antipsychotic agents are $\mathrm{D}_{2}$ receptor antagonists that exert their effect through blocking $\mathrm{D}_{2}$ receptors in the mesolimbic pathway. The catecholamine dopamine is thought to play a key role in the pathophysiology of psychotic disorders (7). Particularly, the mesolimbic dopamine pathway is involved in the emergence of psychotic symptoms. However, in addition to the mesolimbic pathway, dopamine is also the main neurotransmitter in the nigrostriatal and tuberoinfundibular circuit. The latter projects to the pituitary gland, where dopamine acts as a neuroendocrine inhibitor of prolactin secretion. $\mathrm{D}_{2}$ receptor blockade on lactotrophe cells by classic antipsychotics is therefore a potential mechanism explaining antipsychoticinduced hyperprolactinaemia. Aripiprazole has a high affinity for $\mathrm{D}_{2}$ and $\mathrm{D}_{3}$ dopamine receptors and also for 5HT-7 serotonin receptors. However, in a cell-type-specific manner, aripiprazole has partial dopamine agonist activity as well (8). This location-dependent adaptive functionality is described as 'functional selectivity'. Aripiprazole acts as a dopamine agonist under conditions of low endogenous dopamine activity (8), such as on the lactotrophe cells 
of the anterior pituitary gland. Thus, while acting as a $\mathrm{D}_{2}$ receptor blocking antipsychotic in the mesolimbic dopamine pathway, it has dopamine receptor agonist properties in the tuberoinfundibular pathway and thereby inhibits prolactin secretion.

A number of case reports of patients with prolactinoma and concomitant psychiatric disease who were successfully treated with aripiprazole have been published in the psychiatric literature $(9,10)$. Recently, a case report on the successful suppression of prolactin levels by aripiprazole in a woman with a microprolactinoma and cabergoline-induced mania has been published (11). Apart from this case, there are, to our knowledge, no other reports in the endocrinological literature up to now. Our case also illustrates that a reduction in prolactinoma size of comparable proportion as observed during treatment with the classic dopaminergic drugs such as bromocriptine, quinagolide or cabergoline can also be observed during aripiprazole treatment.

In conclusion more than 10-year follow-up of a woman with psychotic symptoms and a microprolactinoma strongly suggests that aripiprazole is capable of ameliorating both clinical problems: psychosis and hyperprolactinaemia. This finding could be clinically relevant for patients with psychotic symptoms and hyperprolactinaemia. Treatment with aripiprazole could be beneficial in similar cases.

\section{Declaration of interest}

The authors declare that there is no conflict of interest that could be perceived as prejudicing the impartiality of the research reported.

\section{Funding}

This research did not receive any specific grant from any funding agency in the public, commercial or not-for-profit sector.

\section{Patient consent}

Written informed consent was obtained from the patient for publication of this case report.
Author contribution statement

Ilse Bakker reviewed all patient data and the relevant literature and drafted the manuscript. Both co-authors have contributed towards patient care, management and also towards writing and finalising the draft.

\section{References}

1 Klibanski A 2010 Prolactinomas. New England Journal of Medicine 362 1219-1226. (doi:10.1056/NEJMcp0912025)

2 Prague JK, Ward CL, Mustafa OG, Whitelaw BC, King A, Thomas NW \& Gilbert J 2014 Delayed presentation of late-onset cerebrospinal fluid rhinorrhoea following dopamine agonist therapy for giant prolactinoma. Endocrinology, Diabetes \& Metabolism Case Reports. 2014 140020. (doi:10.1530/EDM-14-0020)

3 Konopelska S, Quinkler M, Strasburger CJ \& Ventz M 2008 Difficulties in the medical treatment of prolactinoma in a patient with schizophrenia - a case report with a review of the literature. Journal of Clinical Psychopharmacology 28 120-122. (doi:10.1097/ jcp.0b013e3181603f8f)

4 Inoue A, Miki S, Seto M, Kikuchi T, Morita S, Ueda H, Misu Y \& Nakata Y 1997 Aripiprazole, a novel antipsychotic drug, inhibits quinpirole-evoked GTPase activity but does not up-regulate dopamine D2 receptor following repeated treatment in the rat striatum. European Journal of Pharmacology 321 105-111.

5 Li X, Tang Y \& Wang C 2013 Adjunctive aripiprazole versus placebo for antipsychotic-induced hyperprolactinaemia: meta-analysis of randomized controlled trials. PLOS ONE 8 e70179. (doi:10.1371/ journal.pone.0070179)

6 Howes OD \& Kapur S 2009 The dopamine hypothesis of schizophrenia: version III - the final common pathway. Schizophrenia Bulletin 35 549-562. (doi:10.1093/schbul/sbp006)

7 Burris KD, Molski TF, Xu C, Ryan E, Tottori K, Kikuchi T, Yocca FD \& Molinoff PB 2002 Aripiprazole, a novel antipsychotic, is a highaffinity partial agonist at human dopamine D2 receptors. Journal of Pharmacology and Experimental Therapeutics 302 381-389.

8 Shapiro DA, Renock S, Arrington E, Chiodo LA, Liu LX, Sibley DR, Roth BL \& Mailman R 2003 Aripiprazole, a novel atypical antipsychotic drug with a unique and robust pharmacology. Neuropsychopharmacology 28 1400-1411. (doi:10.1038/sj.npp.1300203)

9 Sheldrick AJ \& Grunder G 2008 Aripiprazole reduces serum prolactin in a woman with prolactinoma and acute psychosis. Pharmacopsychiatry 41 160. (doi:10.1055/s-2008-1076721)

10 Freeman B \& Levy W 2007 Successful monotherapy treatment with aripiprazole in a patient with schizophrenia and prolactinoma. Journal of Psychiatric Practice 13 120-122. (doi:10.1097/01. pra.0000265771.47153.a0)

11 Burback L 2015 Management of a microprolactinoma with aripiprazole in a woman with cabergoline-induced mania. Endocrinology, Diabetes \& Metabolism Case Reports 2015 EDM150100. (doi:10.1530/EDM-15-0100)

Received in final form 4 May 2016

Accepted 13 may 2016 